\section{Psicología IBEROAMERICANA}

\section{Psicología Iberoamericana}

ISSN: 1405-0943

psicología.iberoamericana@uia.mx

Universidad Iberoamericana, Ciudad de México

México

González Rivera, Ilse; Díaz-Loving, Rolando

Desarrollo y análisis psicométrico del Inventario de Indefensión Aprendida en la Pareja

Psicología Iberoamericana, vol. 23, núm. 1, enero-junio, 2015, pp. 58-65

Universidad Iberoamericana, Ciudad de México

Distrito Federal, México

Disponible en: http://www.redalyc.org/articulo.oa?id=133944230007

- Cómo citar el artículo

- Número completo

- Más información del artículo

Página de la revista en redalyc.org

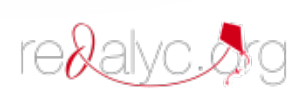

Sistema de Información Científica

Red de Revistas Científicas de América Latina, el Caribe, España y Portugal Proyecto académico sin fines de lucro, desarrollado bajo la iniciativa de acceso abierto 


\title{
Desarrollo y análisis psicométrico del Inventario de Indefensión Aprendida en la Pareja
}

\author{
Psychometric analysis and development of inventory \\ of acquired defenselessness in couples
}

Ilse González Rivera y Rolando Díaz-Loving ${ }^{1}$

Universidad Nacional Autónoma de México

\section{RESUMEN}

La indefensión aprendida se define como el estado psicológico que se produce cuando un individuo no puede con trolar ni predecir los acontecimientos que configuran su vida, lo que provoca perturbaciones motivacionales, cognoscitivas y emocionales. La indefensión puede presentarse de forma generalizada o en aspectos específicos de la vida de una persona. Esta investigación explora la indefensión aprendida en el contexto de las relaciones de pareja. La muestra se conformó con 280 mexicanos de entre 20 y 50 años de edad que se encuentran en una relación de pareja y que cohabitan en ella. Se les aplicó el instrumento de indefensión aprendida en la pareja construido y validado para este estudio; la escala de autoeficacia (Cid, Orellana y Barriga, 2010); el Inventario Multifacético de Satisfacción Marital (Ojeda, Díaz-Loving y Rivera Aragón 1997); y la Escala de Autoevaluación de la Depresión (García, 2002). Se realizaron análisis psicométricos y estadísticos con el objetivo de obtener evidencias de validez de criterio, de constructo y de divergencia y convergencia, así como de confiabilidad del instrumento para población mexicana. Los análisis muestran propiedades psicométricas adecuadas.

Palabras clave: indefensión aprendida; parejas; mexicanos; satisfacción marital; depresión

\section{ABSTRACT}

Acquired defenselessness is defined as a psychological state produced when individuals cannot control or predict events that shape their lives, causing motivational, learning, and emotional distress. Defenselessness can manifest itself in a general way, or in specific aspects of a person's life. This paper explores acquired defenselessness in the context of relations between couples. The same consisted of 280 Mexicans aged between 20 and 50 in a cohabitational relationship. The instrument was applied for defenselessness acquired in the relationship, constructed and validated for this study: the self-efficacy scale (Cid, Orellana and Barriga, 2010); the multifaceted inventory of marital satisfaction (Ojeda, Díaz-Loving and Rivera Aragón 1997); and the depression self-assessment scale (García, 2002). Psychometric and statistical analyses were carried out in order to ascertain validity of criteria, construct, divergence and convergence, as well as the reliability of the instrument for the Mexican population. The analysis demonstrated adequate psychometric properties.

Keywords: acquire defenselessness, couples, mexicans, marital satisfaction, depression.

Fecha de recepción: 4 de junio de 2014 - Fecha de aceptación: 14 de noviembre de 2014

${ }^{1}$ Contacto: Ilse González. Tel: 56222326. Correo electrónico: glez.psi@gmail.com. Av. Universidad 3004, Edificio D, Cubículo 8, Copilco Universidad, Coyoacán, México, Distrito Federal. 
Cuando las personas se encuentran frente a condiciones de su vida que son percibidas como aversivas e incontrolables, es decir que no hay contingencia entre sus actos y lo que sucede en su entorno, aprenden a que sus conductas no resuelven las problemáticas de la situación y por lo tanto se encuentran indefensos, a este fenómeno se le denomina indefensión aprendida (Girardi Inmaculada \& Díaz-Loving, 1988). De esta forma, la indefensión aprendida se puede definir como el estado psicológico que puede producirse cuando un individuo no puede controlar ni predecir los acontecimientos que configuran su vida y como consecuencia del cual se originan una serie de perturbaciones motivacionales, cognoscitivas y emocionales (Bernaola, 2007). De hecho, los individuos que presentan indefensión aprendida no perciben claramente la relación entre su comportamiento y las consecuencias que éste produce, lo que los lleva a emitir conductas de pasividad y a la creencia de que nada de lo que hagan puede mejorar su situación (Vinaccia, Contreras, Restrepo, Cadena \& Anaya, 2005) de modo que perciben las situaciones como incontrolables. Aunque existe una tendencia a que esto ocurra de manera generalizada, la indefensión puede ser producida por situaciones específicas, tales como la relación de pareja.

La historia de la pareja comienza cuando dos personas deciden unirse para conformar un nuevo sistema, lo cual lograrán mediante cambios en la asociación entre ellos y con los demás, conformando así un aprendizaje en donde, mediante ensayos y errores aprenderán cómo convivir (Díaz-Loving y Sánchez, 2004). De esta manera las parejas se pueden entender como el efecto de las interacciones, eventos inmediatos y condiciones causales. Estas condiciones implican dos elementos que son 1) las características personales y 2) el ambiente (Levinger, 2000). El ambiente o contexto comprende el conjunto de valores naturales, sociales y culturales existentes en un lugar y un momento determinado (Triandis, 1994). Así, se desarrollan reglas establecidas por el ecosistema sociocultural que determinan el desarrollo de los rasgos, valores, creencias, actitudes y capacidades individuales (DíazLoving, 1996).

En México se han realizado pocos estudios sobre indefensión aprendida en humanos. Ejemplo de estas investigaciones es la realizada por Girardi (1988), que presentó un modelo de incapacidad aprendida que incluye incontrolabilidad, atribución y depresión para adolescentes. En éste, se presenta el control que tienen los sujetos en distintas situaciones cotidianas como las escolares, las relaciones heterosexuales, la familia y las situaciones sociales. Sin embargo, este fenómeno no se ha estudiado específicamente en las relaciones de pareja, por ello el objetivo de este estudio es construir una escala válida, confiable y culturalmente sensible que mida la indefensión aprendida en la pareja.

\section{MÉTODO}

\section{Participantes}

Se conformó una muestra no probabilística accidental de 280 personas, 131 hombres y 149 mujeres con un rango de edad entre 20 y 50 años residentes del Distrito Federal, que se encuentren actualmente en una relación de pareja con una media de 10.4 años y una desviación estándar de 9.8 años. Adicionalmente, el $54.6 \%$ estaban casados y el $45 \%$ en unión libre. Del total de la muestra, el $35.4 \%$ no tenía hijos al momento de responder el instrumento, el $21.8 \%$ tenía un hijo, el $31.4 \%$, dos hijos, el $13.9 \%$ tres hijos, el 5.4\% cuatro hijos y .7\% más de cuatro hijos. El $71.8 \%$ trabajaba en el momento de responder el instrumento, el $15.4 \%$ se dedicaban al hogar y el 8.6\% eran estudiantes. El 3.6\% tenía escolaridad primaria, $18.9 \%$ secundaria, el $37.5 \%$ preparatoria, el $31.8 \%$ licenciatura y el $7.5 \%$ posgrado.

\section{Instrumentos}

- Escala de indefensión aprendida en la pareja: Se diseñó el instrumento de indefensión aprendida en la pareja con la realización de un estudio exploratorio inicial en el cual se preguntó a diez hombres y diez mujeres cuáles eran las cosas que controlaban de su relación y cuáles no controlaban pero que les gustaría hacerlo. Con base en las respuestas se construyeron 45 reactivos del instrumento que se presentaron en formato tipo Likert de cinco puntos.

- Escala de autoeficacia general de Schwarzer y Jerusalem (1995) validada al español en Chile por Cid, Orellana y Barriga, (2010): Se conforma de 10 
reactivos con un puntaje mínimo de diez puntos y un máximo de cuarenta puntos. Las respuestas son tipo Likert de cinco puntos.

- Inventario multifacético de satisfacción marital de Ojeda, Díaz-Loving y Rivera Aragón (1997): Con alfas de Cronbach mayores a .86; compuesto por cuarenta y una afirmaciones presentadas en una escala tipo Likert de cinco puntos que abarcan desde totalmente en desacuerdo hasta totalmente de acuerdo; constituido por seis factores: el físico-sexual, interacción, comunicación y diversión, familia, toma de decisiones e hijos.

- Escala de autoevaluación de la depresión: validada por García (2002), con un alfa de Cronbach de .80; compuesto por 20 reactivos presentados en forma de aseveraciones, la mitad de las cuales son enunciados sintomáticamente positivos y la otra mitad sintomáticamente negativos; con cinco alternativas de respuesta.

\section{Procedimiento}

Se aplicó la batería de instrumentos a la muestra descrita en lugares públicos, en sus casas así como en lugares de trabajo. Se les pidió a los sujetos que contestaran los cuestionarios de forma anónima y se les explicó que sus respuestas formarían parte de un estudio sólo con fines de investigación. Los cuestionarios se entregaron a cada persona dentro de una carpeta para garantizar la confidencialidad de las respuestas. Una vez recolectados los datos, se procedió a hacer el análisis estadístico, para lo cual se utilizó el paquete estadístico SPSS 15.

Con los datos obtenidos se obtuvieron medidas de dispersión y tendencia central con el fin de describir de manera general el patrón de respuesta de los participantes. Se llevaron a cabo los pasos del procedimiento estadístico para la validación del instrumento de indefensión aprendida y se obtuvieron los datos psicométricos de los demás instrumentos. Una vez asegurados los parámetros de validez y confiabilidad de todas las medidas obtenidas se procedió a hacer las correlaciones entre los instrumentos para obtener la validez del instrumento.

\section{RESULTADOS}

Capacidad de discriminación de cada reactivo. Para obtener indicadores del funcionamiento de cada reactivo se formó una variable sumando los puntajes del total de los reactivos y se formaron grupos de puntajes altos y bajos con base en los cuartiles de esa variable. Posteriormente se compararon los promedios de cada reactivo entre los grupos altos y bajos mediante la prueba $t$ de Student. Diferencias estadísticamente significativas entre ambos grupos denotarían que el reactivo puede discriminar a quienes reportan puntajes altos o bajos en el total de la escala. Dieciséis de los cuarenta y cinco reactivos no obtuvieron buenos resultados. Estos reactivos no fueron incluidos en los análisis posteriores.

Validez de constructo. Se obtuvieron evidencias de la validez de constructo del instrumento sometiendo los 29 reactivos que obtuvieron buenos resultados en el procedimiento anterior a un análisis factorial de componentes principales con normalización de Kaiser y rotación ortogonal Varimax. Resultaron tres factores, que explican el 58.09\% de la varianza, con un alfa de Cronbach total de .75. Tal como se muestra en la Tabla 1.

Tabla 1. Estructura factorial del instrumento de indefensión aprendida.

\begin{tabular}{|c|c|c|c|}
\hline Reactivos & Control pareja & Control propio & Control externo \\
\hline Siempre debo pedir la opinión de mi pareja. & .662 & & \\
\hline Mi pareja lleva las riendas de la familia. & .597 & & \\
\hline Mi pareja decide la hora en la que debo llegar a casa. & .592 & & \\
\hline Mi pareja decide cuándo salir de vacaciones. & .558 & & \\
\hline
\end{tabular}




\begin{tabular}{|c|c|c|c|}
\hline Reactivos & Control pareja & Control propio & Control externo \\
\hline Debo comportarme como a mi pareja le gusta frente a la familia. & .551 & & \\
\hline Mi pareja controla mi vida. & .542 & & \\
\hline $\begin{array}{l}\text { Debo consultar a mi pareja acerca de si se puede gastar } \\
\text { dinero extra. }\end{array}$ & .528 & & \\
\hline Mi pareja me dice cómo actuar frente a los amigos. & .510 & & \\
\hline $\begin{array}{l}\text { Me gustaría poder opinar más acerca de las actividades que realizo } \\
\text { con mi pareja. }\end{array}$ & .493 & & \\
\hline Tengo gran habilidad para corregir a mi pareja cuando se equivoca. & & .637 & \\
\hline Pongo fin a las discusiones con mi pareja. & & .632 & \\
\hline Puedo elegir los temas de conversación con mi pareja. & & .568 & \\
\hline Pudo elegir qué se comerá en la casa. & & .544 & \\
\hline Puedo resolver los problemas que se presentan en la familia. & & .523 & \\
\hline Puedo tomar las decisiones respecto a mi trabajo. & & .517 & \\
\hline Puedo decidir con quién pasaré mi tiempo libre. & & .479 & \\
\hline Tengo habilidad para la administración del dinero. & & .425 & \\
\hline Puedo aconsejar a mis hijos cuando tienen problemas. & & .414 & \\
\hline Siempre puedo decidir qué hacer en mi tiempo libre. & & .396 & \\
\hline La crianza de los hijos está fuera de mis manos. & & & .724 \\
\hline Soy incapaz de enseñar valores a mi familia. & & & .590 \\
\hline Las reglas de mi relación las establece mi pareja. & & & .579 \\
\hline Soy incapaz de arreglar los problemas con mi pareja. & & & .569 \\
\hline Alguien más toma las decisiones importantes dentro de mi familia. & & & .547 \\
\hline Soy incapaz de satisfacer mis gustos y necesidades. & & & .540 \\
\hline Soy incapaz de establecer las reglas del hogar. & & & .510 \\
\hline Mi pareja puede decidir quiénes serán mis amigos. & & & .442 \\
\hline Mi pareja decide cuándo debo mostrarle cariño. & & & .437 \\
\hline
\end{tabular}


Validez de criterio. Se obtuvieron indicadores de validez de criterio al correlacionar los factores de la escala de indefensión aprendida en la pareja y la escala de autoeficacia percibida. Como se esperaba correlacionaron de manera estadísticamente significativa y negativa, como se puede ver en la Tabla 2.

Otra evidencia de validez de criterio se obtuvo al realizar las correlaciones entre los factores de la escala de indefensión aprendida en la pareja y la depresión, mediante la que se obtuvieron correlaciones estadísticamente significativas y positivas entre ambas variables, como se muestra en la Tabla 3.

Para continuar con las evidencias de criterio de la escala se obtuvieron correlaciones entre los factores de la escala de indefensión aprendida en la pareja y el inventario de satisfacción marital; los resultados se muestran en la Tabla 4.

Tabla 2. Correlaciones entre la escala de indefensión aprendida en la pareja y la escala de autoeficacia percibida.

\begin{tabular}{c|c} 
Indefensión & Autoeficacia \\
\hline Control de la pareja & -.073 \\
\hline Control propio & $.451\left(^{* *}\right)$ \\
\hline Control externo & $-.177\left(^{* *}\right)$ \\
\hline
\end{tabular}

Nota: ${ }^{*}<0.05 *{ }^{*} \mathrm{p}<0.01$

Tabla 3. Correlaciones entre la escala de Indefensión aprendida en la pareja y depresión.

\begin{tabular}{c|c|c|c} 
Indefensión & Optimismo & Somatización & Tristeza \\
\hline Control de la pareja & $\left.-.268^{* *}\right)$ & $.270\left(^{* *}\right)$ & $.206(* *)$ \\
\hline Control propio & $.387\left(^{* *}\right)$ & $\left.-.203^{* *}\right)$ & $-.371\left(^{* *}\right)$ \\
\hline Control externo & $-328\left(^{* *}\right)$ & $\left..280^{* *}\right)$ & $.219(* *)$ \\
\hline
\end{tabular}

Nota: ${ }^{*}<0.05{ }^{*}{ }^{*} \mathrm{p}<0.01$

Tabla 4. Correlaciones entre la escala de indefensión aprendida en la pareja y el inventario multifacético de satisfacción marital.

\begin{tabular}{c|c|c|c|c}
\hline Indefensión & Comunicación e interacción & Físico-sexual & Toma de decisiones e hijos & Familia \\
\hline Control de la pareja & $-.123\left(^{*}\right)$ & $-.147\left(^{*}\right)$ & -.004 & -.089 \\
\hline Control propio & $.343\left({ }^{* *}\right)$ & $.298(* *)$ & $212(* *)$ & $.237(* *)$ \\
\hline Control externo & $-.222\left(^{* *}\right)$ & $.233(* *)$ & $-.139(*)$ & $-.225\left(^{* *}\right)$ \\
\hline
\end{tabular}

Nota: ${ }^{*}<0.05{ }^{*} \mathrm{p}<0.01$ 


\section{DISCUSIÓN}

Los análisis de capacidad de discriminación muestran propiedades psicométricas adecuadas para los reactivos que conforman la escala de indefensión aprendida en la pareja. Los resultados de confiabilidad obtenidos a través de los análisis de consistencia interna indican que la escala es confiable. Se eliminaron los reactivos que mostraron propiedades psicométricas inadecuadas en los análisis de capacidad de discriminación, pues estas afirmaciones tienen significados diferentes para los participantes o no están relacionadas con el fenómeno de la indefensión aprendida.

Respecto a la manera en que se formaron los factores de la escala, si bien se compone de un factor que indica indefensión aprendida y uno que indica control, la existencia de un tercer factor denominado "control externo" resulta discutible. Las afirmaciones que componen a este factor se refieren a falta de control sobre la vida marital y de familia, pero en éste no se percibe que sea la pareja quien tiene el control sino como algo externo sin especificar. Se puede concluir que se refiere a la interferencia de terceros en la relaciones de pareja, tales como la familia extendida. Puesto que México es un país que le da mucha importancia al grupo y la familia (Díaz-Guerrero, 1972), las afirmaciones que aquí se presentan pueden referirse a personas externas (como podría ser la suegra, otros miembros de la familia e incluso los vecinos) que posiblemente llevan el control o influyen en ciertos aspectos de la vida de pareja, lo cual suele ser común en este país.

Las correlaciones que dan pie a la validez de criterio fueron congruentes con lo esperado teóricamente y con lo reportado en estudios previos. Tal como se esperaba se encontró una correlación negativa entre los factores de la indefensión aprendida y la autoeficacia percibida, mientras que estos mismos factores se relacionaron de manera positiva con los factores de la escala de depresión. (Vinaccia, Cadena, Contreras, Juárez \& Anaya, 2004). Específicamente, los resultados muestran que cuando existe un mayor control de parte de la pareja, es decir, cuando un miembro tiene que pedir siempre la opinión del otro, se presenta menos optimismo, más somatización y tristeza, los cuales son síntomas de la depresión. Estos resultados denotan la importancia de la investigación sobre la indefensión aprendida en el contexto marital, pues tiene impli- caciones en la salud de los individuos y se relaciona directamente con la depresión, que tiene repercusiones en la salud física y mental, de modo que afecta las relaciones interpersonales, la motivación para actuar en su vida y en la realización como individuos (Yescas, Ascencio, Vargas, Barbosa \& Lugo, 2008).

Cuando un individuo percibe que las decisiones respecto al hogar, la crianza de los hijos, la vida en pareja y el esparcimiento es algo que puede controlar, modificar e incluso mejorar, entonces se muestra más optimista respecto a la vida, menos triste, se propone metas y siente que es capaz de lograrlas. La ausencia de indefensión aprendida en la vida de pareja tiene repercusiones positivas en su vida personal.

En cuanto a la relación que se obtuvo de los factores de la escala de indefensión aprendida en la pareja y los factores de la escala de satisfacción marital se puede notar cómo el percibir falta de control dentro de la relación afecta negativamente la satisfacción en todos los factores de la escala, mientras que el percibir control propio en la relación afecta positivamente a la satisfacción marital en todos los factores, sobre todo en la comunicación e interacción con la pareja y en el físico y sexual (Rhyne, 1981). Las correlaciones de estas dos escalas proporcionan evidencias de la validez de la escala de indefensión. Sin embargo, debido a que la escala se enfoca al contexto marital, se propone que se profundice más al respecto al vincularlo con componentes de las relaciones de pareja, tales como los estilos de amor y la felicidad.

Es interesante analizar los resultados en el contexto de la cultura mexicana, pues los roles que desempeñan los miembros de la familia mexicana dependen íntimamente de las proposiciones socioculturales estipuladas. En México aún prevalece la premisa del machismo y la abnegación de las mujeres, de modo que se concibe el rol de ellas como cuidadoras del hogar y de los hijos y el de los hombres como proveedores y dominantes (Díaz Guerrero, 1972).

En este estudio, la mayor parte de la muestra trabaja, tanto hombres como mujeres, sin embargo puede que muchas mujeres funjan como amas de casa después del trabajo, por lo que ellas podrían sentir indefensión cuando perciben falta de control en lo referente al cuidado de los hijos y la limpieza del hogar, mientras 
que los hombres percibirían ausencia de control en lo relacionado con cuestiones monetarias y sexuales. Sin embargo, en los últimos años ha habido cambios en el papel de la mujer dentro de la sociedad mexicana tales como que el que se sienten menos sujetas a la autoridad del hombre, quieren independizarse más, tener oportunidades de trabajo externas, buscar oportunidades profesionales y, en general, ser menos dóciles respecto al varón (Díaz-Guerrero \& Balderas-González, 2000), por lo que se propone estudiar estas diferencias en hombres y mujeres en los factores de la escala, pues cada factor está compuesto por reactivos que se relacionan tanto con cuestiones del hogar como con cuestiones monetarias y de pareja.

Cuando los mexicanos se encuentran ante una situación de estrés tienden a evitarla a toda costa pues para ellos aceptar y aguantar pasivamente el estrés se percibe como la mejor manera de sobrellevarlo (Díaz Guerrero, 1972). La sumisión, la dependencia, la cortesía, el aguante y la "concha" podrían ser considerados como virtudes socioculturales mexicanas o como formas realísticas de confrontación de los estreses de la vida, por lo que el estar indefensos puede ser una característica cultural constante.

La religión predominante en México refuerza las premisas de que "éste es un valle de lágrimas", y del deber adaptarse fácil a la tragedia y a la muerte, por lo

\section{REFERENCIAS}

Bernaola, L. (2007). Estudio correlacional entre estilos de crianza e indefensión aprendida en estudiantes del quinto y sexto grado de primaria de la Institución Educativa No 2013. Asociación Policial. Tesis de Licenciatura en Enfermería. Lima: Universidad Nacional Mayor de San Marcos.

Cid, P.H., Orellana, A. \& Barriga, O. (2010). "Validación de la escala de autoeficacia general en Chile". Revista Médica de Chile, 138, 551-557.

Díaz Guerrero, R. (1972). Hacia una teoría históricobio-psico-socio-cultural del comportamiento humano. México: Trillas.

Díaz Guerrero, R. (1994). Psicología del mexicano: descubrimiento de la etnopsicología. México: Trillas. que son comunes expresiones como "nada puede hacerse" y "no hay salida" ante situaciones que parecen escapar de su control (Díaz Guerrero, 1994). Debido a lo anterior se propone hacer una exploración más a fondo sobre cómo se percibe y desarrolla el fenómeno de la indefensión en mexicanos y en comparación con otras culturas colectivistas e individualistas (Díaz Guerrero, 1972).

Otro factor que puede influir en los resultados se relaciona con las características de la muestra. El nivel socioeconómico puede estar influyendo ya que la incapacidad para acceder a los recursos económicos y culturales disponibles provoca que a las personas les resulte más difícil controlar y predecir el ambiente provocando apatía, indiferencia y depresión por lo que se propone hacer comparaciones en niveles socioeconómicos y con escolaridades diferentes.

Finalmente, se puede concluir que, si bien, la escala de indefensión aprendida en la pareja muestra características psicométricas adecuadas y se obtuvieron evidencias de confiabilidad y validez de la misma, este estudio es una primera aproximación a la indefensión aprendida en la pareja, con las limitaciones mencionadas anteriormente, pero estableciendo una propuesta para próximas investigaciones al enfatizar la importancia de estudiar más el fenómeno pues tiene repercusiones en la vida personal, familiar y de pareja. •

Díaz-Guerrero, R. \& Balderas-González (2000). “Amor versus el poder: el nuevo primer factor de la filosofía de la vida." La psicología Social en México, 8, 134-142.

Díaz-Loving, R. (1996). Una teoría bio-psico-socio-cultural de la relación de pareja. Revista de Psicología Contemporánea. 2(1). 18-29.

Díaz-Loving, R. \& Sánchez, R. (2004). Psicología del amor: Una visión integral de la relación de pareja. México: Miguel Ángel Porrúa.

García, M. (2002). Asociación de la satisfacción marital, la evitación del conflicto y depresión. Tesis de Maestría. México: UNAM.

Girardi Inmaculada, C. (1998). Un modelo de incapacidad aprendida para adolescentes mexicanos. Tesis de Doctorado. México: Facultad de Psicología. UNAM. 
Girardi Inmaculada, C. \& Díaz-Loving, R. (1988). "Validez de constructo del inventario de incapacidad aprendida", Salud mental, 11(3), 40-47.

Levinger, G (2000). "Las relaciones cercanas: tres ingredientes centrales." Revista de Psicología Social y Personalidad. XVI (1), 43-64.

Ojeda, A., Díaz-Loving, R \& Rivera Aragón, S. (1997). “El doble vínculo como determinante de la satisfacción marital.” Revista de Psicología Social y Personalidad, 13(1), 19-40.

Rhyne, D. (1981). "Bases of marital satisfaction among men and women." Journal of marriage and family, 43(4), 941-955.

Rivera, A (1992). Atracción interpersonal y su relación con la satisfacción marital y la relación ante la interacción de pareja. Tesis de Maestría. México: Facultad de Psicología. UNAM.

Schwarzer, R. \& Jerusalem, W. (1995). "Generalized Self-efficacy Scale. In: J. Weinman, J. Wright \& M. Johnston (1995).2 Measures in health psychology:
A user's portfolio. Causal and control beliefs. Windsor: England, 35-37.

Triandis, H.C. (1994). Culture and social Behavior. New York: McGraw-Hill.

Vinaccia, S., Cadena, J., Contreras, F., Juárez, F. \& Anaya, J.M. (2004). "Relaciones entre variables sociodemográficas, incapacidad funcional, dolor y desesperanza aprendida en pacientes con diagnóstico de artritis reumatoide”. International Journal of Clinical and Health Psychology, 4, 91-103.

Vinaccia, S. Contreras, F. Restrepo L., Cadena, J. \& Anaya, J. (2005). "Autoeficacia, desesperanza aprendida e incapacidad funcional en pacientes con diagnóstico de artritis reumatoide." International Journal of Clinical and Health Psychology, 5(1), 129-142.

Yescas, E., Ascencio, L., Vargas, S., Barbosa, R. \& Lugo, G. (2008). "Depresión, ansiedad y desesperanza aprendida en pacientes con artritis reumatoide" Psicología y Salud, 18(1), 81-89. 